\title{
Optimality of Linear Codes for Broadcast-Mode Multicast Networks
}

\author{
Rathinakumar Appuswamy \\ Dept. of Electrical and Computer Eng. \\ University of California, San Diego \\ La Jolla, CA 92093-0407 \\ Email: rathnam@ucsd.edu
}

\author{
Massimo Franceschetti \\ Dept. of Electrical and Computer Eng. \\ University of California, San Diego \\ La Jolla, CA 92093-0407 \\ Email: massimo@ece.ucsd.edu
}

\author{
Kenneth Zeger \\ Dept. of Electrical and Computer Eng. \\ University of California, San Diego \\ La Jolla, CA 92093-0407 \\ Email: zeger@ucsd.edu
}

\begin{abstract}
It is known that linear codes are sufficient to solve the multicast network coding problem when each out-edge of a network node carries its own specific function of the in-edges of the node, i.e. operating in "point-to-point-mode." Alternatively, in "broadcast-mode," a network has the property that for each node, every out-edge of the node carries the same function of the in-edges of the node. Only one transmission is required in order to send the same function on all of the out-edges of a node. The edge functions in broadcast-mode can vary from node to node and each edge can carry an arbitrary number of transmissions, with at most one per time unit. We prove that linear codes are sufficient, in terms of total number of transmissions, for multicast networks in broadcast-mode. That is, we show that for any broadcast-mode solution to a multicast network, there exists a linear broadcastmode solution over some finite field which does not increase the total number of network transmissions.
\end{abstract}

\section{INTRODUCTION}

One commonly studied network coding model, which we call point-to-point-mode, consists of transmitting along each out-edge of a network node an arbitrary function of the symbols (or vectors of symbols) which are inputs to the node (either messages generated at the node or the contents carried on the in-edges to the node). At each receiver node, a demanded message is constructed as an arbitrary function of the inputs to the receiver. In such a scenario, each edge is used at most once and thus carries at most one symbol (or vector of symbols). A solution then consists of an assignment of edge functions, and decoding functions at the receivers, so that each receiver can recover all of the messages that it demands. Under such a model, a network is solvable if there exists a solution for some alphabet. A solution is called linear if all edge functions and decoding functions are linear over some alphabet. We will restrict attention to linearity over finite fields. It is known [3] that in point-to-point-mode linear codes are sufficient for multicast networks. This is stated in the following lemma.

Lemma I.1. If a multicast network is solvable in point-topoint-mode, then it is linearly solvable over some finite field.

An alternative network coding model, which we call broadcast-mode, was studied in [1], [4], [5]. In this model, when any node makes a transmission, it is assumed that every node adjacent to it via an edge (i.e. its neighbors), receives the transmission. In [5] the measure of performance was the total number of transmissions required to multicast a message from the source to a set of receiver nodes and this was motivated by energy-constrained wireless networks. These authors studied the advantage of network coding over routing (i.e. when no coding is allowed and every node simply relays messages), for some specific networks, and in the special case when every node is the source of a message which is demanded by every other node. (Additional work of the same authors also appears in [2].)

In contrast, the work in [1], [4] did not focus on energy efficiency, but instead concentrated on throughput questions. The work in [1] showed that linear codes are sufficient to achieve capacity in broadcast-mode multicast networks with possible transmission erasures (all erasures are assumed known at all nodes). The work in [4] gave some practical code constructions for this case.

Finally, $\mathrm{Wu}$ et al. [6] considered the problem of energy efficient graph construction for broadcast-mode multicast networks. For an arbitrary node placement, they formulated a linear program to optimally assign the transmission radii of the nodes to construct a graph with a single source that reaches all destination nodes, and uses the minimum energy per transmitted unit of information using networks coding.

In contrast with these previous works, our present paper focuses on the smallest total number of broadcast-mode transmissions required to solve the multicast problem in any given graph, and shows that this number does not increase if one is restricted to use only linear codes.

We now describe our model in more detail. We assume that at any given time step, each node in the network may transmit exactly one message or not transmit at all. Whenever a node transmits a message, all of its out-edges deliver it successfully to the nodes they point to. Furthermore, each node remembers and can use all the messages it has already received at any given time. Whereas point-to-point-mode codes allow at most one transmission per edge, broadcast-mode codes allow the re-use of edges over multiple time units and with possibly different encoding functions. Thus, clearly for any connected network, there always exists a broadcast-mode solution. We measure the energy cost of a given broadcast-mode solution 
by counting the total number of transmissions used to deliver the messages to the receivers demanding them.

In this paper we use Lemma I.1 to prove that linear codes are also sufficient for energy efficient broadcast-mode coding in multicast networks. The gist of our approach will be to show that for any broadcast-mode solution to a multicast network, there exists a linear broadcast-mode solution with an equal number of transmissions, over a sufficiently large finite field.

The proofs of lemmas are omitted due to space limitations.

\section{A. Notation}

A multicast communication network consists of a finite, acyclic, directed multigraph $G$ with a unique source node $s$, that generates a finite set of messages, and whose receiver nodes each demand all of the messages generated by $s$.

Let $\mathcal{V}(G), \mathcal{E}(G)$, and $\mathcal{R}(G)$ denote $G$ 's node set, edge set, and receiver set, respectively. We assume that only one symbol can be transmitted per unit time on each edge of $G$.

The elements of $\mathcal{V}(G)-\mathcal{R}(G)-s$ are called interior nodes. For each node $n \in \mathcal{V}(G)$, let $\mathcal{E}_{i}(G, n)$ and $\mathcal{E}_{o}(G, n)$ be the sets of in-edges and out-edges of $n$, respectively. The integers $\left|\mathcal{E}_{i}(G, n)\right|$ and $\left|\mathcal{E}_{o}(G, n)\right|$ are the in-degree and out-degree of $n$, respectively.

At any given time unit, any edge may carry one symbol from a fixed finite alphabet $\mathcal{A}$. In point-to-point-mode, each edge may only be used during one time unit, whereas in broadcastmode edges may be re-used at different time units.

Each symbol transmitted by an interior node is a function of the symbols it has received on its in-edges. Hence, at certain time steps, interior nodes receive a symbol on each of its inedges and transmit a symbol on their out-edges determined by a function of the incoming symbols (called an encoding function). For each source message, every receiver computes a decoding function to try to deduce the source message. A multicast network is said to be solvable if there exists an assignment of edge functions and decoding functions that allows every receiver demand to be satisfied. In broadcastmode, such an assignment is also a function of time.

Let $\mathcal{T}_{i}$ denote a list of the network nodes that transmit a message at time $i$, and let $\mathcal{F}_{i}$ denote the corresponding list of encoding functions used by the nodes in $\mathcal{T}_{i}$ at time $i$.

Thus, $\left|\mathcal{T}_{i}\right|=\left|\mathcal{F}_{i}\right|$ for all $i$. A transmission scheme over $\tau$ time units is a collection

$$
\left\{\left(\mathcal{T}_{i}, \mathcal{F}_{i}\right): i=1,2, \ldots, \tau\right\}
$$

together with a set of decoding functions at the receiver nodes.

A transmission scheme is a broadcast-mode solution if the collection $\left\{\left(\mathcal{T}_{i}, \mathcal{F}_{i}\right)\right\}$ along with the decoding functions defining the transmission scheme ensure that all the receiver nodes can deduce all the messages generated by the source $s$. We say that a transmission scheme is linear (over a finite field alphabet $\mathcal{A}$ ) if the network encoding functions at each $\mathcal{F}_{i}$, for $i=1, \ldots, t$, and the decoding functions used by all the receiver nodes, are linear over $\mathcal{A}$; otherwise, the transmission scheme is said to be nonlinear. The number of transmissions in a broadcast-mode solution is given by

$$
\sum_{i=1}^{t}\left|\mathcal{T}_{i}\right| .
$$

A broadcast-mode solution is optimal if no other broadcastmode solution can use fewer transmissions. We note that minimizing the number of transmissions is important in various applications such as energy-limited radio networks, where battery energy is constrained.

\section{Optimality of Linear Network CODING FOR BROADCAST-MODE}

In this section, we establish that linear network coding is sufficient to achieve energy efficient transmission in the following sense. Given a broadcast-mode solution with $t$ transmissions to a network $\mathcal{N}$, there exists a linear broadcastmode solution with the same number of transmissions over a large enough finite field.

To prove this result we construct another broadcast-mode network $\mathcal{N}_{1}$, by splitting all of the non-receiver nodes of $\mathcal{N}$, and we show that this network has a broadcast mode solution with twice as many transmissions as required to solve $\mathcal{N}$. Then, we create a solvable multicast point-to-point-mode network $\mathcal{N}_{2}$ from the solution to $\mathcal{N}_{1}$. Since the constructed multicast network $\mathcal{N}_{2}$ has a point-to-point-mode solution, it also has a linear solution by Lemma I.1. Then, we show that this linear point-to-point-mode solution induces a linear broadcast-mode solution on the network $\mathcal{N}_{1}$, and finally a linear broadcast-mode solution on the network $\mathcal{N}$ with exactly the same number of transmissions of the original solution. We next describe these steps more formally.

Definition II.1. For an arbitrary broadcast-mode multicast network $\mathcal{N}$ with graph $G$ and source node s, define a new broadcast-mode multicast network $\mathcal{N}_{1}$ with graph $G_{1}$ and the same messages, demands, and receivers, as follows.

- For every $x \in \mathcal{V}(G)-\mathcal{R}(G)$, the graph $G_{1}$ contains two nodes $x^{\prime}$ and $x^{\prime \prime}$ and an edge from node $x^{\prime}$ to node $x^{\prime \prime}$ (see Figure 1). The in-edges of $x^{\prime}$ and the out-edges of $x^{\prime \prime}$ correspond to the in-edges and out-edges, respectively, of node $x$. Thus,

$$
\begin{aligned}
& \mathcal{V}\left(G_{1}\right)=\mathcal{R}(G) \cup \bigcup_{x \in \mathcal{V}(G)-\mathcal{R}(G)}\left\{x^{\prime}, x^{\prime \prime}\right\} \\
& \mathcal{E}\left(G_{1}\right)=\bigcup_{x \in \mathcal{V}(G)-\mathcal{R}(G)}\left(\left\{\left(x^{\prime}, x^{\prime \prime}\right)\right\}\right. \\
&\left.\cup\left\{\left(x^{\prime \prime}, y^{\prime}\right) \mid(x, y) \in \mathcal{E}(G)\right\}\right) .
\end{aligned}
$$

If the network $\mathcal{N}_{1}$ has a solution $\left\{\left(\mathcal{T}_{i}, \mathcal{F}_{i}\right): i=1, \ldots, \tau\right\}$, then define a new point-to-point-mode multicast network $\mathcal{N}_{2}$ with graph $G_{2}$ and the same messages, demands, and receivers, as $\mathcal{N}$, as follows.

- For each $x \in \mathcal{V}(G)$, let $\sigma(x)$ denote the number of transmissions by the node $x^{\prime \prime} \in \mathcal{V}\left(G_{1}\right)$ in the given broadcast-mode solution. For each $x \in \mathcal{V}(G)$, replicate the node $x^{\prime \prime} \in \mathcal{V}\left(G_{1}\right)$ a total of $\sigma(x)$ times (see Figure 2 ). 
- Suppose $x \in \mathcal{V}(G)$ and $x^{\prime}, x^{\prime \prime} \in \mathcal{T}_{\tau_{1}}, \mathcal{T}_{\tau_{2}}, \ldots, \mathcal{T}_{\tau_{\sigma(x)}}$, Then, a directed edge is added to $G_{2}$ from $x^{\prime}$ to each of the replicas, $x_{1}^{\prime \prime}, \ldots, x_{\sigma(x)}^{\prime \prime}$, of $x^{\prime \prime}$.

- For each $k$, label the edge $\left(x^{\prime}, x_{k}^{\prime \prime}\right)$ in $G_{2}$ by the encoding function $f_{x, k}$ used in $G_{1}$ by nodes $x^{\prime}$ and $x^{\prime \prime}$ at time $\tau_{k}$. Let the out-edges of $x_{k}^{\prime \prime}$ in $G_{2}$ point to the same nodes as the out-edges of $x^{\prime \prime}$ in $G_{1}$. Each out-edge of node $x_{k}^{\prime \prime}$ is labeled the same as the edge $\left(x^{\prime}, x_{k}^{\prime \prime}\right)$.

- Let each receiver in $G_{2}$ have the same decoding functions as the corresponding receiver in $G_{1}$.

Thus,

$$
\begin{gathered}
\mathcal{V}\left(G_{2}\right)=\mathcal{R}\left(G_{1}\right) \cup \bigcup_{i=1}^{\tau} \bigcup_{x^{\prime}, x^{\prime \prime} \in \mathcal{T}_{i}}\left(\left\{x^{\prime}\right\} \cup\left\{x_{k}^{\prime \prime}: 1 \leq k \leq \sigma(x)\right\}\right) \\
\mathcal{E}\left(G_{2}\right)=\bigcup_{x \in \mathcal{V}(G)-\mathcal{R}(G)}\left(\left\{\left(x^{\prime}, x_{k}^{\prime \prime}\right): 1 \leq k \leq \sigma(x)\right\}\right. \\
\left.\cup\left\{\left(x_{k}^{\prime \prime}, y\right):(x, y) \in \mathcal{E}(G)\right)\right\} .
\end{gathered}
$$

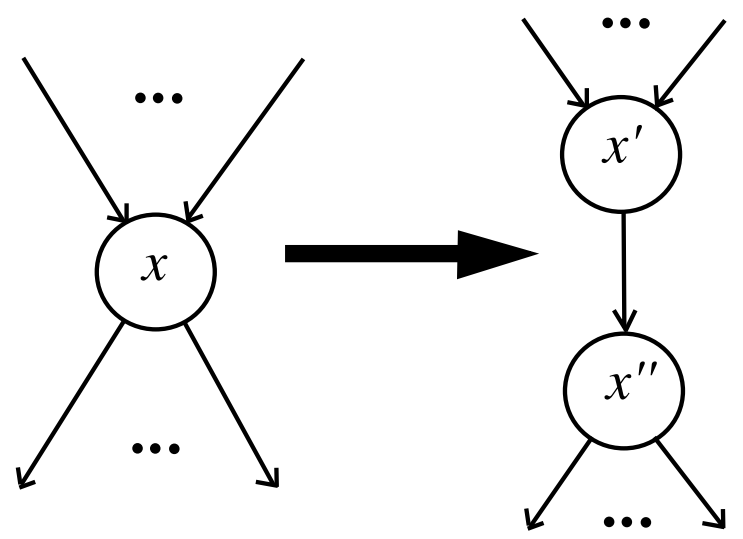

Fig. 1. The splitting of a node $x$ in the network $\mathcal{N}$ into two nodes $x^{\prime}$ and $x^{\prime \prime}$ in the augmented network $\mathcal{N}_{1}$.

The following lemmas refer to the networks $\mathcal{N}_{1}$ and $\mathcal{N}_{2}$ given in Definition II.1 (here we assume that the link between $x^{\prime}$ and the dummy node $x^{\prime \prime}$ is delay free).

Lemma II.2. A multicast network $\mathcal{N}$ has a broadcast-mode solution with $t$ transmissions over $\tau$ time units if and only if the network $\mathcal{N}_{1}$ has a broadcast-mode solution with $2 t$ transmissions over $\tau$ time units.

In the proof of the following lemma, a broadcast-mode solution to $\mathcal{N}_{1}$ with $2 t$ transmissions over $\tau$ time units is used to obtain a point-to-point-mode solution to $\mathcal{N}_{2}$, which in turn (via Lemma I.1) is used to obtain a point-to-point-mode linear solution to $\mathcal{N}_{2}$, which, finally, is used to obtain a broadcastmode linear solution to $\mathcal{N}_{1}$ with $2 t$ transmissions over $t$ time units.

Lemma II.3. For any multicast network $\mathcal{N}$, if the network $\mathcal{N}_{1}$ has a broadcast-mode solution with $2 t$ transmissions, then there exists a finite-field alphabet such that $\mathcal{N}_{1}$ has a

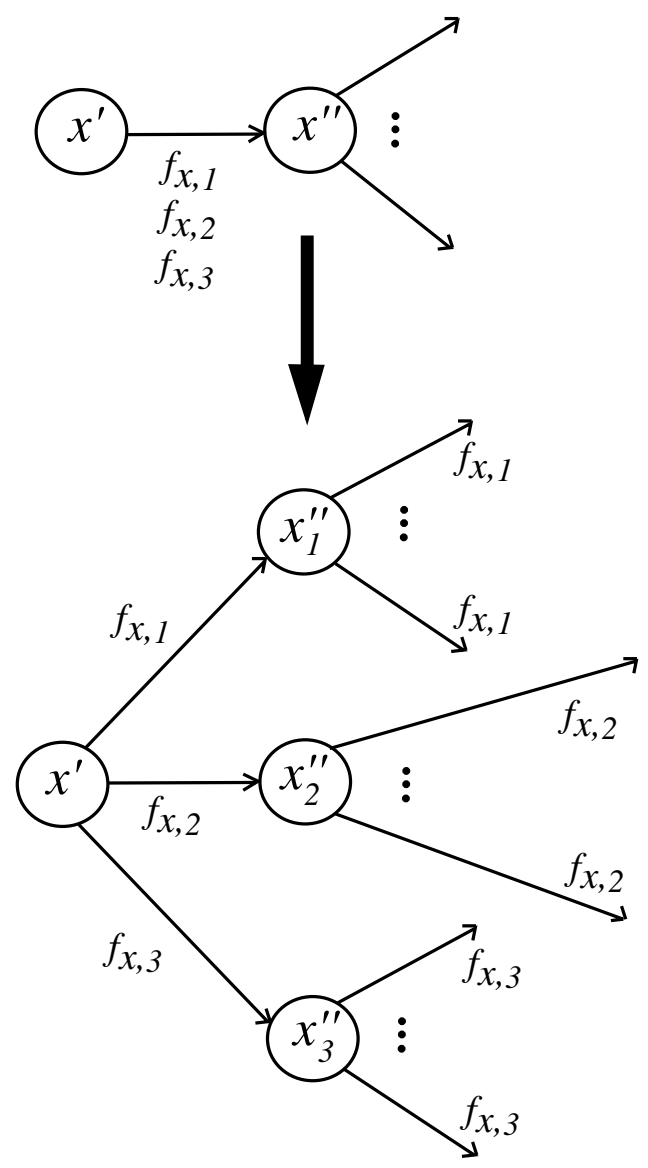

Fig. 2. The splitting of a node $x^{\prime \prime}$ in the network $\mathcal{N}_{1}$ into multiple nodes $x_{1}^{\prime \prime} \ldots x_{\sigma(x)}^{\prime \prime}$ in the new network $\mathcal{N}_{2}$ and corresponding encoding functions assignment. In this example, $\sigma(x)=3$

broadcast-mode linear solution with $2 t$ transmissions over $t$ time units.

The following theorem is the main result of this paper. It establishes the sufficiency of linear coding for multicast networks in broadcast-mode, analogous to the same result for point-to-point mode given in [3].

Theorem II.4. If a multicast network has a broadcast-mode solution with $t$ transmissions, then there exists a finite field alphabet such that the network has a linear broadcast-mode solution with $t$ transmissions over at most $t$ time units.

Proof. Assume that the network $\mathcal{N}$ has a broadcast-mode solution with $t$ transmissions over $\tau$ time units. By Lemma II.2, the network $\mathcal{N}_{1}$ has a broadcast-mode solution with $2 t$ transmissions over $\tau$ time units. By Lemma II.3, the network $\mathcal{N}_{1}$ has a point-to-point-mode linear solution (over some finite field) with $2 t$ transmissions over $t$ time units. Finally, by using Lemma II.2 again, the network $\mathcal{N}$ must have a linear broadcast-mode solution with $t$ transmissions over $t$ time units.

We point out that is not evident from the proof of the theorem above whether it is possible to obtain a linear broadcast- 
mode solution spread over the same number of time units as the given broadcast-mode solution, except in the trivial case when there are no processing nodes (i.e. every node in the network is a relay node). An interesting open question is to determine the smallest number of time units needed to achieve a linear broadcast-mode solution for a solvable multicast network.

\section{ACKNOWLEDGMENT}

This work was supported by the National Science Foundation and the UCSD Center for Wireless Communications.

(Submitted to ISIT06 on January 15, 2006.)

\section{REFERENCES}

[1] A. Dana, R. Gowaikar, R. Palanki, B. Hassibi, M. Effros, "Capacity of wireless erasure networks", Submitted to the IEEE Transactions on Information Theory.

[2] C. Fragouli, J. Widmer, and J.-Y. Le Boudec, "A network coding approach to energy efficient broadcasting: from theory to practice," Infocom, 2006.

[3] S-Y. R. Li, R.W. Yeung, and N. Cai, "Linear network coding," IEEE Transactions on Information Theory, vol. 49, no. 2, pp. 371-381, February 2003.

[4] D. Lun, M. Medard, R. Koetter, M. Effros, "On coding for reliable communication over packet networks." Submitted to IEEE Transactions on Information Theory.

[5] J. Widmer, C. Fragouli, and J.-Y. Le Boudec, "Low-complexity energyefficient broadcasting in wireless ad-hoc networks using network coding," First Network Coding Workshop (NETCOD), Riva del Garda, Italy, 2005".

[6] Y. Wu, P. Chu, S. Kung, "Minimum energy multicast in mobile ad-hoc networks using network coding." IEEE Transactions on Communications, 53(11):1906-1918, Nov. 2005. 